\title{
The effects of psychosis risk variants on brain connectivity:
}

\section{a review}

\author{
Omar Mothersill ${ }^{1,2 \dagger}$, Sinead Kelly ${ }^{1,2 \dagger}$, Emma Jane Rose ${ }^{1,2}$ and Gary Donohoe ${ }^{1,2 *}$ \\ ${ }^{1}$ Neuropsychiatric Genetics Group, Department of Psychiatry, Trinity College Dublin, Dublin, Ireland \\ ${ }^{2}$ Trinity College Institute for Neuroscience, Trinity College Dublin, Dublin, Ireland
}

Edited by:

Daniela Tropea, Trinity College Dublin Ireland

Reviewed by:

Mikhail Pletnikov, Johns Hopkins University, USA

Andrew M. Mclntosh, The University of Edinburgh, UK

\section{${ }^{*}$ Correspondence:}

Gary Donohoe, Associate Professor of Clinical Psychology and

Neuropsychology, Neuropsychiatric

Genetics Group, Department of

Psychiatry, Trinity College Dublin,

Trinity Centre for Health Sciences, St.

James' Hospital, Dublin 8, Ireland.

e-mail:donoghug@tcd.ie

+ Omar Mothersill and Sinead Kelly

have contributed equally to this work.
In light of observed changes in connectivity in schizophrenia and the highly heritable nature of the disease, neural connectivity may serve as an important intermediate phenotype for schizophrenia. However, how individual variants confer altered connectivity and which measure of brain connectivity is more proximal to the underlying genetic architecture (i.e., functional or structural) has not been well delineated. In this review we consider these issues and the relative sensitivity of imaging methodologies to schizophrenia-related changes in connectivity. We searched PubMed for studies considering schizophrenia risk genes AND functional or structural connectivity. Where data was available, summary statistics were used to determine an estimate of effect size (i.e., Cohen's $d$ ). A random-effects meta-analysis was used to consider (1) the largest effect and (2) all significant effects between functional and structural studies. Schizophrenia risk variants involved in neurotransmission, neurodevelopment and myelin function were found to be associated with altered neural connectivity. On average, schizophrenia risk genes had a large effect on functional (mean $d=0.76$ ) and structural connectivity (mean $d=1.04$ ). The examination of the largest effect size indicated that the outcomes of functional and structural studies were comparable $(Q=2.17, p>0.05)$. Conversely, consideration of effect size estimates for all significant effects suggest that reported effect sizes in structural connectivity studies were more variable than in functional connectivity studies, and that there was a significant lack of homogeneity across the modalities $(Q=6.928, p=0.008)$. Given the more variable profile of effect sizes associated with structural connectivity, these data may suggest that structural imaging methods are more sensitive to a wider range of effects, as opposed to functional studies which may only be able to determine large effects. These conclusions are limited by methodological considerations, and require further investigation involving larger samples, multiple genes, and novel analysis techniques for confirmation.

Keywords: schizophrenia, functional connectivity, structural connectivity, genotype, effect size

\section{INTRODUCTION}

\section{EXAMINING THE FUNCTION OF SCHIZOPHRENIA (SZ) RISK VARIANTS}

Schizophrenia is a complex genetic disorder affecting roughly $1 \%$ of the world's population (see Lewis and Lieberman, 2000 for a review). It is characterized by hallucinations and delusions, reduced emotion and cognitive impairment, and imposes a heavy cost on society (for example, the total cost of psychotic disorders in Europe in 2010 was recently calculated as $€ 93.9$ billion; Gustavsson et al., 2011). While there is no consensus about its exact causes, the heritability of SZ is estimated to be about $80 \%$ (Sullivan et al., 2003). Genome-wide association (GWAS) and copy number variation studies have identified several common and rare gene variants associated with the disorder (O'Donovan et al., 2008). Understanding the function of these variants could, therefore, lead to a greater understanding of disease pathogenesis, which could direct new treatments.

Schizophrenia patients present with variable symptom profiles and distinct disease trajectories. This heterogeneity may be in part due to the complex genetics of SZ, which in turn poses significant problems for understanding the mechanisms by which genetic variants confer risk for this disease. In an attempt to address this complexity, researchers have focused on so-called "intermediate phenotypes," which are measurable variations that occur on the pathway between genes and disease, and as such may be closer to the underlying genetic architecture than clinical symptoms (see Gottesman and Gould, 2003 for a review). Possible intermediate phenotypes for SZ include changes in brain chemistry, structure, function (Braff et al., 2007) and connectivity (Meyer-Lindenberg, 2009).

While changes in functional and structural connectivity may be a critical aspect of the $\mathrm{SZ}$ disease profile, there has been little systematic evaluation of the relative sensitivity of these different indices to genetic risk for SZ. In this meta-analysis we outline empirical investigations that have utilized functional magnetic resonance imaging (fMRI) or diffusion tensor imaging (DTI) to investigate the effects of SZ risk variants on functional and structural brain connectivity. We also consider the relative magnitude 
of these effects, in order to determine the extent of the genetic impact on brain connectivity.

\section{ALTERED FUNCTIONAL CONNECTIVITY IN SZ}

In the early 20th century, German neurologist Carl Wernicke proposed that SZ arises from altered neural connectivity (or dysconnectivity) rather than from abnormalities in specific parts of the brain (see Stephan et al., 2009 for a review). One hundred years later, advances in neuroimaging technology have enabled scientists to empirically consider dysconnectivity as a key component of SZ pathogenesis.

Two or more brain regions are said to be functionally connected if they show a correlation of activity over time (Friston et al., 1993). The hypothesis that functional connectivity is altered in SZ is supported by positron emission tomography (PET; e.g., Friston and Frith, 1995; Meyer-Lindenberg et al., 2001, 2005) and fMRI studies (Lawrie et al., 2002), which reveal abnormal prefrontotemporal connectivity in SZ patients while they perform cognitive tasks. Electroencephalogram (EEG) research also demonstrates abnormal functional connectivity patterns in patients with SZ (Breakspear et al., 2003), and one genetic mouse model of SZ reveals decreased hippocampal-prefrontal connectivity during a T-maze task (Sigurdsson et al., 2010).

Support for the role of functional connectivity as an intermediate phenotype for psychiatric disorders includes a fMRI study by Pezawas et al., 2005). These authors examined the effects of a 5-HTTLPRpolymorphism that is associated with anxiety and depression, on functional connectivity between the amygdala and cingulate cortex. It was reported that variant-associated changes in connectivity predicted almost $30 \%$ of the variance in the behavioral effects of this polymorphism. Behavioral variability was also in fact better predicted by changes in connectivity than changes in regional brain activation.

Understanding the underlying biological causes of altered functional connectivity has the potential to lead to a better understanding of SZ pathogenesis, but so far the etiology of functional dysconnectivity remains unclear. However, different mechanisms have been proposed, which we will discuss in the following sections.

\section{THE “DISCONNECTION" HYPOTHESIS OF SZ}

The "disconnection" hypothesis was first proposed by Karl Friston and colleagues in the 1990s (Friston, 1998). This hypothesis postulates that SZ is primarily caused by abnormal $N$-methyl-Daspartate (NMDA)-receptor mediated synaptic plasticity, which in turn, is caused by dysregulation of these receptors by neurotransmitters such as dopamine. Support for the role of the NMDA receptor in SZ comes from several studies. Firstly, drugs that block the NMDA-receptor, such as ketamine and phencyclidine, can induce psychotic symptoms in healthy controls (see Javitt, 2010 for a review). Similarly, ketamine administration induces sensory processing deficits in controls similar to deficits seen in patients, suggesting a role for NMDA receptors in these deficits (Umbricht et al., 2000). Activity of midbrain dopaminergic neurons is partially regulated by glutamatergic projections from the prefrontal cortex (PFC), acting via NMDA receptors, and NMDA receptor-blockade enhances amphetamine-induced increases in striatal dopamine in controls, similar to increases seen in SZ patients (Kegeles et al., 2000). Finally, genetic variants that play a role in NMDA-signaling have been associated with increased SZ risk in candidate gene studies [e.g., G72,GRM3 and RGS4; see Harrison and Weinberger (2005) for a review].

\section{FUNCTIONAL CONNECTIVITY MRI ANALYSIS TECHNIQUES}

Functional connectivity can be measured with a variety of tools (e.g., PET, EEG), but this review will focus on papers using fMRI to measure the phenotype in healthy controls and patients with SZ. Using the blood oxygen level dependent (BOLD) response as an indirect measure of neuronal activity (Ogawa et al., 1990), there are a range of approaches to analysis. This review will focus on seeded connectivity and psychophysiological interaction (PPI).

A seeded connectivity analysis begins with the selection of a seed region, which can be a voxel, or cluster of voxels in the fMRI time-series (Nallasamy and Tsao, 2011). The mean time-course for the seed region is then correlated with all other voxels in the brain. Voxels that pass a certain threshold are considered to be functionally connected with the seed region, resulting in a functional connectivity map. While the PPI approach also measures the co-variation of the BOLD signal in voxels across the brain (Friston et al., 1997), it also measures changes in the interactions between brain regions in response to different psychological tasks.

\section{STRUCTURAL CONNECTIVITY AND SZ}

White matter (WM) contains myelinated nerve cells that connect various gray matter (GM) areas of the brain to each other, and carry nerve impulses between neurons. Compromised WM integrity is evident in SZ (Kubicki et al., 2007; Ellison-Wright and Bullmore, 2009). Moreover, WM abnormalities are apparent in individuals at high risk of SZ and also in patients during the early stages of illness, suggesting that these abnormalities may be a stable characteristic of the disease (Witthaus et al., 2008; Perez-Iglesias et al., 2010). There are two key postulations regarding the nature of WM deficits in SZ: The "global theory" and the "macro-circuit theory." The global theory of WM disruption in SZ suggests that WM is compromised uniformly throughout the brain, whereas the macro-circuit theory proposes that specific WM tracts are compromised, which may be a cause or consequence of abnormalities in the gray matter regions these tracts connect (Buchsbaum et al., 2006; Konrad and Winterer, 2008).

\section{Diffusion tensor imaging}

Diffusion tensor imaging is a method used to measure the diffusion of water molecules in brain WM. Healthy brain WM has a complex axonal structure and, therefore, water diffusion will be restricted along the direction of the axons. This is known as anisotropic diffusion. However, if brain WM is compromised water diffusion can become less restricted (i.e., isotropic). A common measure derived from DTI to describe the degree of anisotropy during diffusion is fractional anisotropy (FA). However, other measures of diffusion such as radial and axial diffusivity can also be obtained. Based on the voxel-wise information provided by DTI, fiber tracking algorithms can be implemented in regions of interest to reconstruct the underlying three-dimensional WM pathways. While caution must be exercised when interpreting measures of anisotropy (Jones, 2008, 2010; Tournier et al., 2011), such measures are thought to index structural integrity of WM tracts and, 
thus, may be reasonably considered to be implicit indices of brain connectivity.

\section{WM integrity and SZ: evidence from DTI investigations}

A review by Kubicki et al. (2007) noted that the most frequent positive findings of DTI studies in SZ were decreased FA within the prefrontal and temporal lobes, as well as abnormalities within the fiber bundles connecting these regions. WM tracts within these regions that were found to be affected included: (a) the uncinate fasciculus that connects parts of the limbic system with areas in the frontal cortex; (b) the cingulum bundle; and (c) the arcuate fasciculus that connects part of the temporo-parietal junction with the frontal cortex and is thought to be part of the superior longitudinal fasciculus. Ellison-Wright and Bullmore (2009) conducted a meta-analysis of 15 DTI studies, which included a total of 407 patients with SZ and 383 comparison subjects. Results identified two regions of FA decreases in SZ subjects in comparison to controls. The first region was in the left frontal deep WM, which is traversed by WM tracts interconnecting the frontal lobe, thalamus and cingulate gyrus. The tracts include: (a) anterior thalamic radiation (ATR); (b) corticobulbar tracts; (c) inter-hemispheric fibers running through the genu of the corpus callosum; (d) the inferior fronto-occipital fasciculus; (e) the cingulum bundle. The second region was in the left temporal deep WM that is traversed by WM tracts interconnecting the frontal lobe, insula, hippocampus-amygdala, temporal and occipital lobes. These tracts include: (a) inter-hemispheric fibers running through the splenium of the corpus callosum; (b) the inferior fronto-occipital fasciculus; (c) the inferior longitudinal fasciculus; (d) the fornix/striaterminalis. These two reviews of the current DTI/SZ literature suggest that specific networks of WM are disrupted in SZ providing support for the macro-circuit theory of WM disruption in the disease.

\section{Pathophysiological mechanisms of compromised WM}

As the integrity of axons is dependent on myelination and factors influencing myelination, it is possible that myelin and oligodendroglia function also plays a role in the pathophysiology of SZ (Davis et al., 2003). Since myelination also impacts synaptic plasticity, oligodendrocyte abnormality and subsequent myelin dysfunction may contribute to the development of SZ by altering synaptic function and information processing (Fields, 2008). Conduction velocity along axons is also thought to be essential for learning processes (Fields, 2008) and disruption of this has the potential to lead to the range of cognitive impairments observed in SZ (Tanaka et al., 2009). Furthermore, oligodendrocyte and myelin dysfunction also impacts neuronal activity that is relevant to SZ, such as glutamate and dopamine signaling. Evidence from psychotic episodes of multiple sclerosis (MS) patients and experimentally induced demyelination suggests that altered myelin function leads to altered dopamine signaling (Takahashi et al., 2011). Similar analyses have also revealed increased levels of glutamate in brains of MS patients as well as increased expression of glutamate receptors on oligodendrocytes (Takahashi et al., 2011). Glutamate transporters are also present on oligodendroglia and are thought to regulate glutamate concentrations to prevent glutamate-induced excitotoxicity (Pitt et al., 2003). Over activation of oligodendroglial glutamate receptors is excitotoxic and can result in oligodendrocyte death (Davis et al., 2003).

Recently, attention has turned toward the consideration of genes that influence oligodendrocyte architecture and how these genes may also be associated with SZ risk. Hakak et al. (2001) examined the expression of 6500 genes derived from postmortem cortical tissue of SZ patients and controls. The expression levels of six myelin-related genes were significantly down regulated for SZ patients in comparison to control subjects. These genes included: myelin-associated glycoprotein (MAG), CNP, myelin and lymphocyte protein (MAL), gelosn (GSN), ErbB3, and transferring. Down regulation of these genes supports the view that oligodendrocytes, the cell type from which all these genes derive in the brain, contribute to the pathophysiology of SZ.

\section{USING IMAGING GENETICS TO EXAMINE NEURAL CONNECTIVITY}

Two previous meta-analyses have considered the magnitude of the impact of gene variants on brain function, each reporting large effect sizes. Munafo et al. (2008) examined the effect sizes of the 5-HTTLPR polymorphism and amygdala activation, while Mier et al. (2010) examined the magnitude of effect of the catechol-Omethyltransferase (COMT) Val158Met polymorphism on brain function, reporting association between this variant and activation of the PFC. However, to our knowledge no studies have specifically considered the effect size of gene variants in studies of functional and structural connectivity, or compared effect sizes between these phenotypes. Consideration of the relative impact of these two measures of brain connectivity will help us to better delineate whether or not one phenotype is more proximal to the underlying genetics, and thus preferential as an intermediate phenotype for studies of SZ. This could not only aid our theoretical understanding of the SZ disease trajectory but may also have significant practical implications for future investigations.

\section{METHODS}

We searched for relevant papers based on the criteria of studies that included genes implicated in SZ risk and measures of either DTI or functional connectivity. PubMed (http://www.ncbi.nlm.nih.gov/pubmed/) was used to search for relevant functional and structural connectivity papers published until June 2011. The following search terms were included in this search: [schizophrenia OR schiz*] AND [genetic or gene*] AND [MRI OR DTI] AND [connectivity] AND [structural OR functional]. This literature search was supplemented with a review of the references from each of the papers identified. In total 24 studies meeting these search criteria were retrieved, including 12 DTI studies and 12 functional connectivity studies. Individual studies differed slightly in terms of MRI acquisition and analysis parameters (e.g., voxel size, size of Gaussian function used for smoothing). However, all studies were included regardless of these differences, due to the small number of studies available. Where the data presented were insufficient for effect size calculations, a request for supplementary data was sent to the corresponding author. This led to data being available for 19 out of the 24 studies identified (10 DTI studies and 9 functional connectivity studies).

Effect size calculations were performed using two online effect size calculators http://www.uccs.edu/ faculty/lbecker/ and 
www.lyonsmorris.com/ma1/index.cfm Estimates of effect size were calculated based upon either descriptive data (i.e., mean, SD, and $N$ ), or statistical data (i.e., $t, F$ ). The purpose of this paper was to estimate differences in effect size rather than differences in direction of effect. That is we were interested in delineating the relative sensitivity of these two indices of brain connectivity to genetic variability, rather than accounting for the overall impact of a specific variant or group of variants. Therefore, direction of effect was not included in the analysis and all effect sizes were considered positive.

A random effects meta-analyses considering the relative difference in the impact of SZ risk genes on functional and structural connectivity was carried out using the comprehensive meta analysis (CMA; software package v2; www.meta-analysis.com). For the purposes of this analysis, Hedge's $g$ and its associated variance were calculated for the outcome of each significant effect in each study. As with prior estimates of Cohen's d, g was calculated using a variety of input variables including descriptive and inferential statistics. In the first analysis, the largest effect for each study was chosen so to reflect the maximal sensitivity to gene effects within each investigation. In a secondary analysis, all of the effects for each significant result in each paper were taken into account. This strategy allowed us to account for both variability in the number and range of significant effects reported across methodologies.

\section{RESULTS}

Overall, $8 \mathrm{fMRI}$ and 10 DTI studies were included in the metaanalysis. Summary information from all of these studies is presented in Tables 1 and 2 .

\section{FUNCTIONAL CONNECTIVITY}

A total of 44 effect sizes were calculated from the functional connectivity studies. Effect sizes (i.e., Cohen's $d$ ) ranged from medium to large ( $d=0.46-1.65)$ with an average effect size of 0.76 $(\mathrm{SD} \pm 0.23)$. The largest effect size $(d=1.65)$ was reported for the impact of a single nucleotide polymorphism (SNP) in ZNF804A on functional connectivity within the right PFC in SZ patients (Rasetti et al., 2011). While large effect sizes $(d>0.7)$ were also calculated in other studies examining the effects of this SNP on functional connectivity (Esslinger et al., 2009, 2011; Rasetti et al., 2011) these results were not consistent: the smallest effect size was also reported for this SNP ( $d=0.46$; Paulus et al., 2011).

\section{STRUCTURAL CONNECTIVITY}

A total of 24 effect sizes were calculated for structural connectivity investigations. Effect sizes ranged from small to large $(d=0.38-$ $1.95)$ with an average effect size of $1.04(\mathrm{SD}=0.42)$. The largest effect size was revealed for the impact of NRG1 SNP on WM integrity in the left ATR (Sprooten et al., 2009). Large effect sizes were also observed for all the other studies examining the impact of NRG1 on WM integrity (all $d>0.80$ ). Similar effect sizes were revealed for studies investigating the ErbB4 gene, with Cohen's $d$ for these studies ranging from 0.81 to 1.41 . Both the MTHFR gene and the 5-HTT gene had large effect sizes of 1.29 and 0.92 respectively. The smallest effect size of 0.38 was computed for the effect of a COMT haplotype on left prefrontal WM integrity. Cohen's d for the COMT papers ranged from 0.38 to 0.76 .

\section{META-ANALYSIS}

In our first meta-analysis we considered only the largest effect sizes in each study (Figure 1; Table 3). This analysis revealed no significant difference in outcome variability between the effect sizes for functional and structural studies $(Q=2.171, p=0.141)$. Our second analysis examined all of the effects for each result in each paper (Figure 2; Table 3). This analysis revealed a significant difference between effects sizes in functional and structural studies $(Q=6.928, p=0.008)$.

\section{DISCUSSION}

The aim of this review was to consider the nature and magnitude of effect of SZ risk variants on functional and structural connectivity. Our focus was the overall magnitude of such effects, rather than delineating the direction of effect of a specific variant. Therefore, we focused on the size, rather than the direction of individuals results. Examining the effect of risk variants on connectivity in fMRI and DTI studies, we found that variation in genes implicated in neurotransmission, plasticity, development and myelin function are associated with altered neural connectivity. Meta-analyses of effect size data revealed that there was no significant difference between the effect sizes of functional and structural studies when the largest effect size of each study was analyzed. However, when all effects were taken into consideration, the effect sizes for the structural studies were larger than in the functional connectivity studies, and there was a significant lack of homogeneity across the modalities. Mostly likely, given the absence of difference when only the largest effects from each set of studies are considered, this difference reflects the greater variation in effect sizes in structural studies compared to fMRI studies.

\section{FUNCTIONAL CONNECTIVITY STUDIES}

To date, the effects on functional connectivity of a number of candidate SZ genes (DISC1, PRODH, PPP1R1B) and one gene with genome-wide significance for SZ risk (ZNF804A), each of which were found to be associated with altered functional connectivity. The mean effect size for the functional connectivity studies was large $(d=0.76)$, with the largest effect size reported for the impact of the rs1344706 variant in ZNF804A.The ZNF804A risk variant rs 1344706 has become the focus of much interest in SZ research over the last 3 years, as GWAS and follow-up analyses have established strong evidence for a link between this variant and risk for the disorder (O'Donovan et al., 2008). While the function of the ZNF804A gene is unknown, it has been speculated to play a role in gene regulation (O'Donovan et al., 2008) and glutamate and dopamine transmission (Esslinger et al., 2009). ZNF804A represents the only SZ-implicated gene that we are aware of whose effects on functional connectivity have been investigated now on a number of occasions. Importantly, the size of effect reported for this variant has varied considerably. For example, as well as having the highest effect of the variants considered it also has the lowest effect of the variants considered ( $d=0.46$; Paulus et al., 2011), suggesting that establishing the true effect of any variant on functional connectivity will require investigation in multiple and adequately powered cohorts. Variants from the candidate SZ gene literature by comparison, while each showing large effects on functional connectivity ( $d$-range: $0.73-0.87$ ), have each only been the subject of 
Table 1 | Details of the functional connectivity studies included in this meta-analysis.

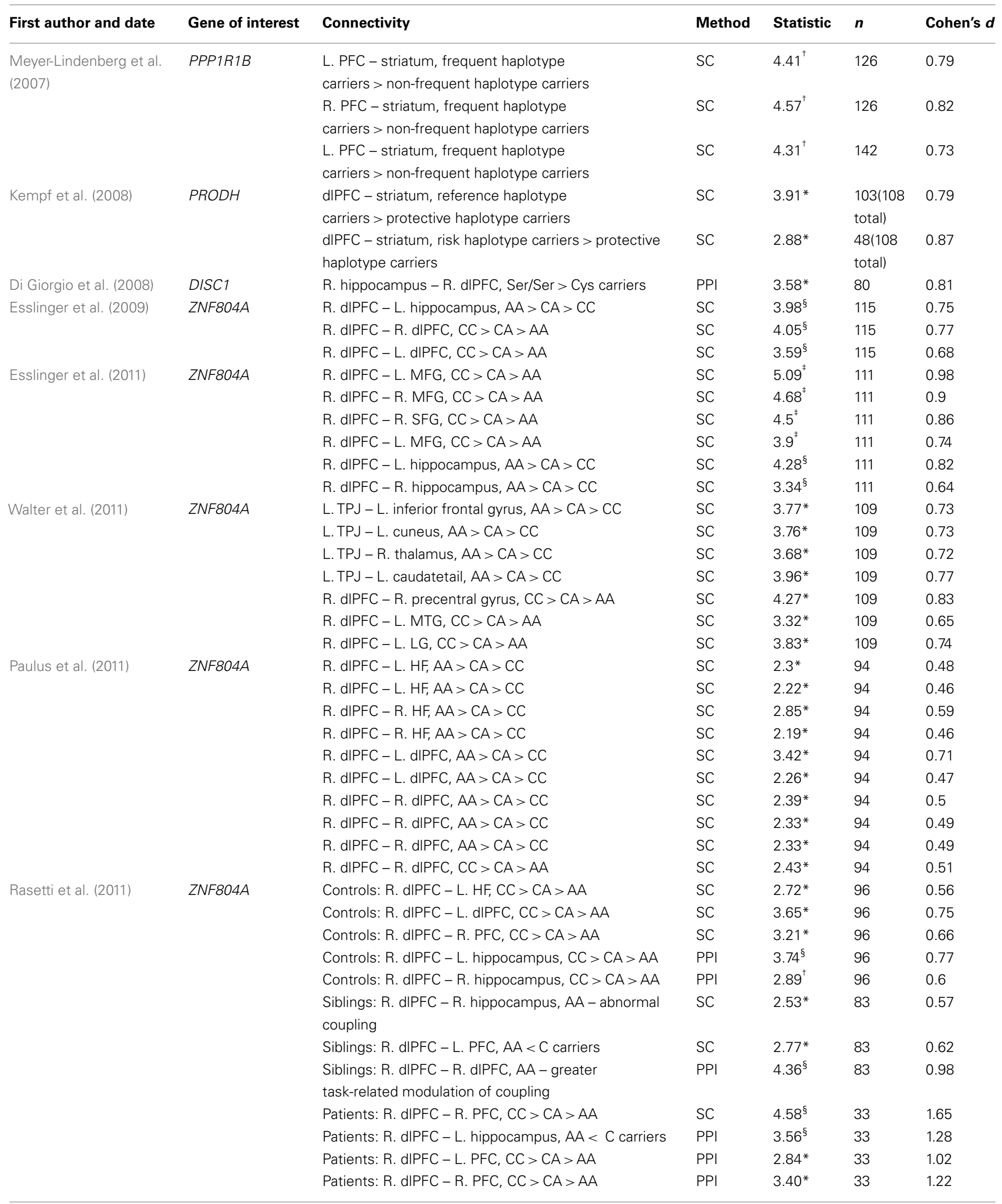

n, sample size; SC, seeded connectivity; PPI, psychophysiological interaction; dIPFC, dorsolateral prefrontal cortex; MFG, middle frontal gyrus; SFG, superior frontal gyrus; TPJ, temporo-parietal junction; MTG, middle temporal gyrus; $L G$, lingual gyrus; IFG, inferior frontal gyrus; * $p$-value is uncorrected for multiple comparisons; ${ }^{\dagger}$ false discovery rate corrected within region of interest; ${ }^{\ddagger}$ false discovery rate corrected for whole brain; ${ }^{5}$ family wise error corrected within region of interest. 
Table 2 | Details of the structural connectivity studies using DTI included in this meta-analysis.

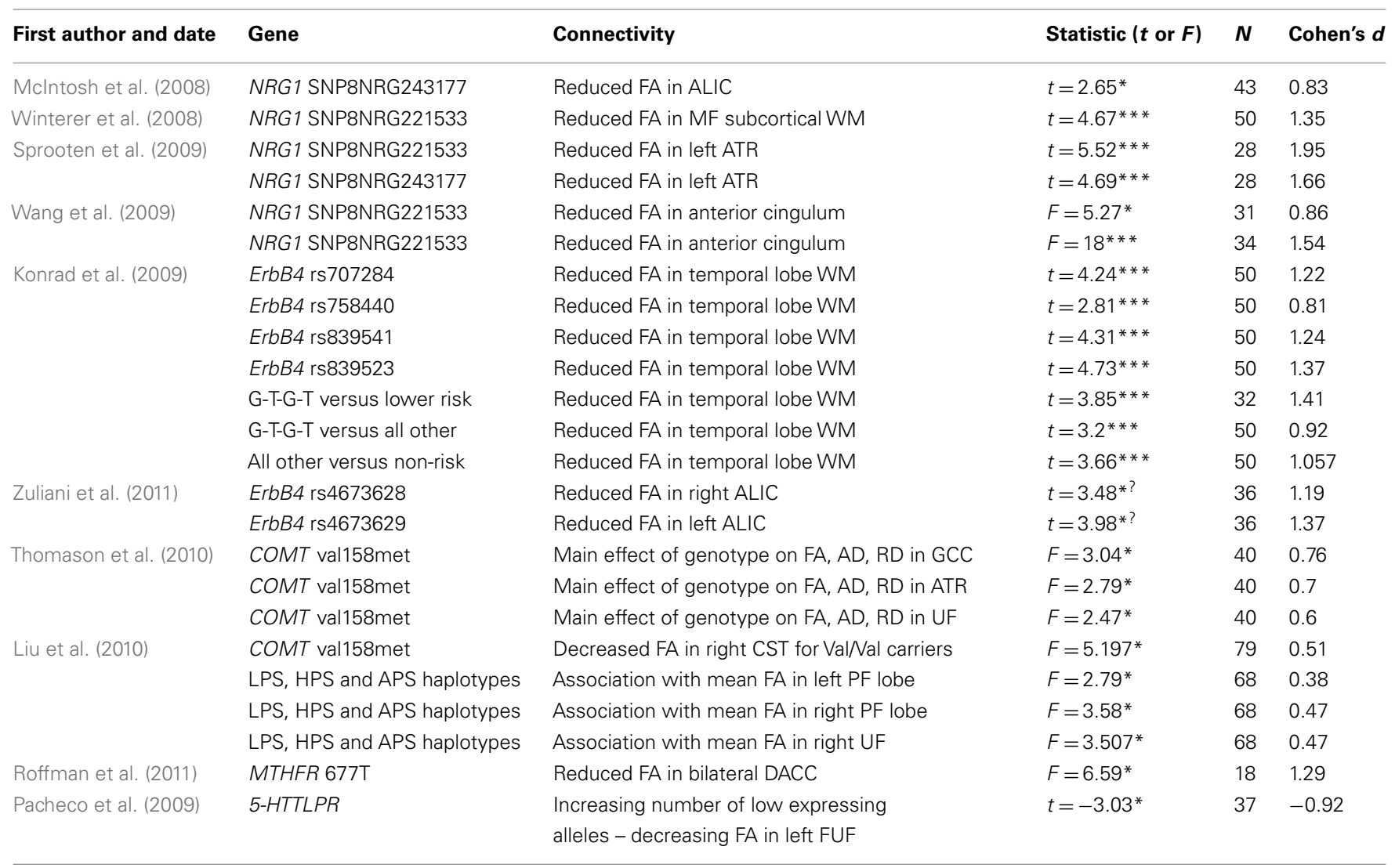

n, sample size; FA, fractional anisotropy; MF, medial frontal; WM, white matter; $A L I C$, anterior limb of internal capsule; $A D$, axial diffusivity; RD, radial diffusivity; ATR,

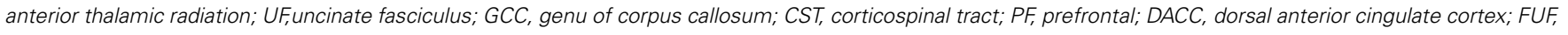
frontal uncinate fasciculus; ${ }^{*} p<0.05 ;{ }^{*}{ }^{*} p<0.001 ;{ }^{*} p<0.05$ family wise error corrected

single studies to date (DISC1; Di Giorgio et al., 2008; PRODH; Kempf et al., 2008; PPP1R1B; Meyer-Lindenberg et al., 2007).

In 10 of the $12 \mathrm{fMRI}$ studies included in this review, SZ risk variants were reported to affect functional circuits that included the PFC during the performance of a variety of tasks, such as memory encoding and retrieval, working memory, emotion processing, and during rest. These findings reflect the PFC's dominant role in many processes related to higher cognitive functioning, making it consistently implicated in SZ pathogenesis (Callicott et al., 2000).There are several possibilities for why PFC function is altered in SZ as reflected in these studies. For example, the "reduced neuropil hypothesis" of schizophrenia suggests that reduced PFC gray matter (observed in the absence of a concomitant change in cell numbers)may reflect decreased dendritic spines/axon terminals; the inefficiencies synaptic transmission expected to result may well lead to the altered functional connectivity patterns seen here (Selemon and Goldman-Rakic, 1999).

\section{STRUCTURAL CONNECTIVITY STUDIES}

A number of candidate SZ risk genes have been investigated in terms of their effects on structural connectivity. This includes genes that are involved in myelination (NRG1, ErbB4) neurotransmission (COMT, MTHFR, 5-HTTLPR) and neurodevelopment
(BDNF and DISC1). Almost all variants considered here were associated with significant variation in FA scores using DTI.

The average effect size for these studies was large, with the largest effect size computed for the impact of NRG1 on FA in the left ATR (Sprooten et al., 2009). The NRG1 gene codes for the $N R G 1$ protein, that is involved in growth and differentiation of neuronal and glial cells and is necessary for the normal development of the nervous system. ErbB4 is a receptor for the NRG1 protein. It is thought that NRG1 may mediate its effects on SZ susceptibility through functional interaction with ErbB4 (Norton et al., 2006). Interestingly, ErbB4 was also observed here to show effects on WM integrity that would be considered to be in of large magnitude. The role of these genes in myelin function suggests a mechanism by which they confer risk for SZ. The relatively large impact of these genes on structural connectivity, noted here, suggests that genetically mediated "dysconnectivity" in SZ results from macro-circuit WM abnormalities in addition to micro-circuit synaptic plasticity. However, since oligodendrocyte dysfunction may also impact synaptic function and information processing via a myelin-dependent impact on synaptic plasticity (Fields, 2008), it remains to be established if the influence of these variants is specific to structural connectivity or if they also impact upon functional connectivity. 


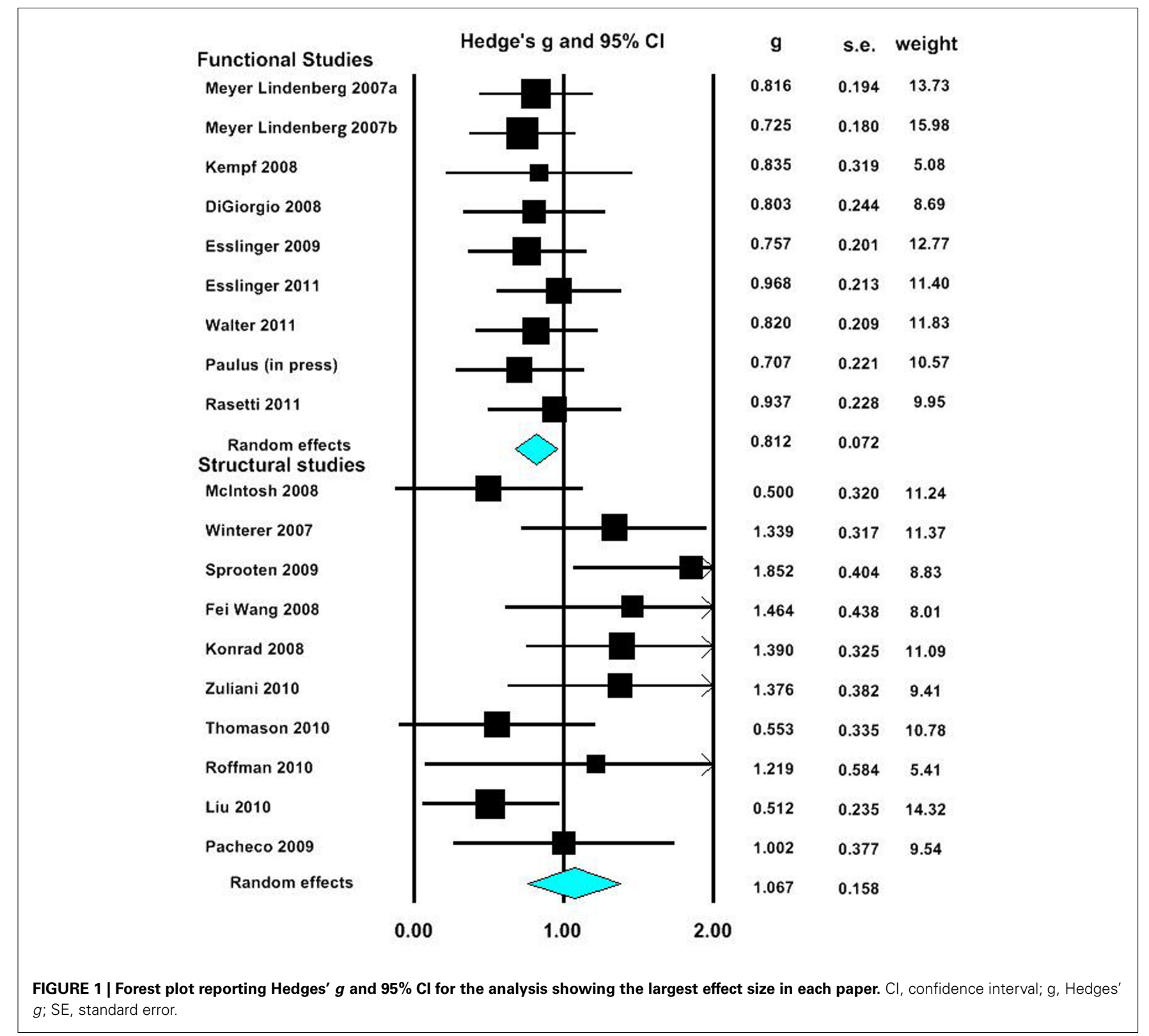

Table 3 | Results of random-effects meta-analysis comparing the relative difference in the impact of variants on functional and structural connectivity.

Effect size and $95 \%$ confidence interval

Total between

\section{ALL ESTIMATES}

Functional

24

Structural

Total between

\begin{tabular}{|c|c|c|c|c|c|c|c|c|c|}
\hline & \multicolumn{6}{|c|}{ Effect size and $95 \%$ confidence interval } & \multicolumn{3}{|c|}{ Heterogeneity } \\
\hline & No. of studies & Point estimate & SE & Variance & Lower limit & Upper limit & $Q$-value & $\mathrm{d} f(Q)$ & $p$-value \\
\hline \multicolumn{10}{|c|}{ MAXIMUM ESTIMATE } \\
\hline Functional & 9 & 0.812 & 0.072 & 0.005 & 0.671 & 0.953 & & & \\
\hline Structural & 10 & 1.067 & 0.158 & 0.025 & 0.758 & 0.377 & & & \\
\hline Total between & & & & & & & 2.171 & 1 & 0.141 \\
\hline Functional & 44 & 0.687 & 0.032 & 0.001 & 0.625 & 0.750 & & & \\
\hline Structural & 24 & 0.934 & 0.088 & 0.008 & 0.761 & 1.108 & & & \\
\hline Total between & & & & & & & 6.928 & 1 & 0.008 \\
\hline
\end{tabular}

\begin{tabular}{|c|c|c|c|c|c|c|c|c|c|}
\hline & \multicolumn{6}{|c|}{ Effect size and $95 \%$ confidence interval } & \multicolumn{3}{|c|}{ Heterogeneity } \\
\hline & No. of studies & Point estimate & SE & Variance & Lower limit & Upper limit & $Q$-value & $\mathrm{d} f(Q)$ & $p$-value \\
\hline \multicolumn{10}{|c|}{ MAXIMUM ESTIMATE } \\
\hline Functional & 9 & 0.812 & 0.072 & 0.005 & 0.671 & 0.953 & & & \\
\hline Structural & 10 & 1.067 & 0.158 & 0.025 & 0.758 & 0.377 & & & \\
\hline Total between & & & & & & & 2.171 & 1 & 0.141 \\
\hline Functional & 44 & 0.687 & 0.032 & 0.001 & 0.625 & 0.750 & & & \\
\hline Structural & 24 & 0.934 & 0.088 & 0.008 & 0.761 & 1.108 & & & \\
\hline Total between & & & & & & & 6.928 & 1 & 0.008 \\
\hline
\end{tabular}

\begin{tabular}{|c|c|c|c|c|c|c|c|c|c|}
\hline & \multicolumn{6}{|c|}{ Effect size and $95 \%$ confidence interval } & \multicolumn{3}{|c|}{ Heterogeneity } \\
\hline & No. of studies & Point estimate & SE & Variance & Lower limit & Upper limit & $Q$-value & $\mathrm{d} f(Q)$ & $p$-value \\
\hline \multicolumn{10}{|c|}{ MAXIMUM ESTIMATE } \\
\hline Functional & 9 & 0.812 & 0.072 & 0.005 & 0.671 & 0.953 & & & \\
\hline Structural & 10 & 1.067 & 0.158 & 0.025 & 0.758 & 0.377 & & & \\
\hline Total between & & & & & & & 2.171 & 1 & 0.141 \\
\hline Functional & 44 & 0.687 & 0.032 & 0.001 & 0.625 & 0.750 & & & \\
\hline Structural & 24 & 0.934 & 0.088 & 0.008 & 0.761 & 1.108 & & & \\
\hline Total between & & & & & & & 6.928 & 1 & 0.008 \\
\hline
\end{tabular}

Functional Studies

Meyer Lindenberg 2007 


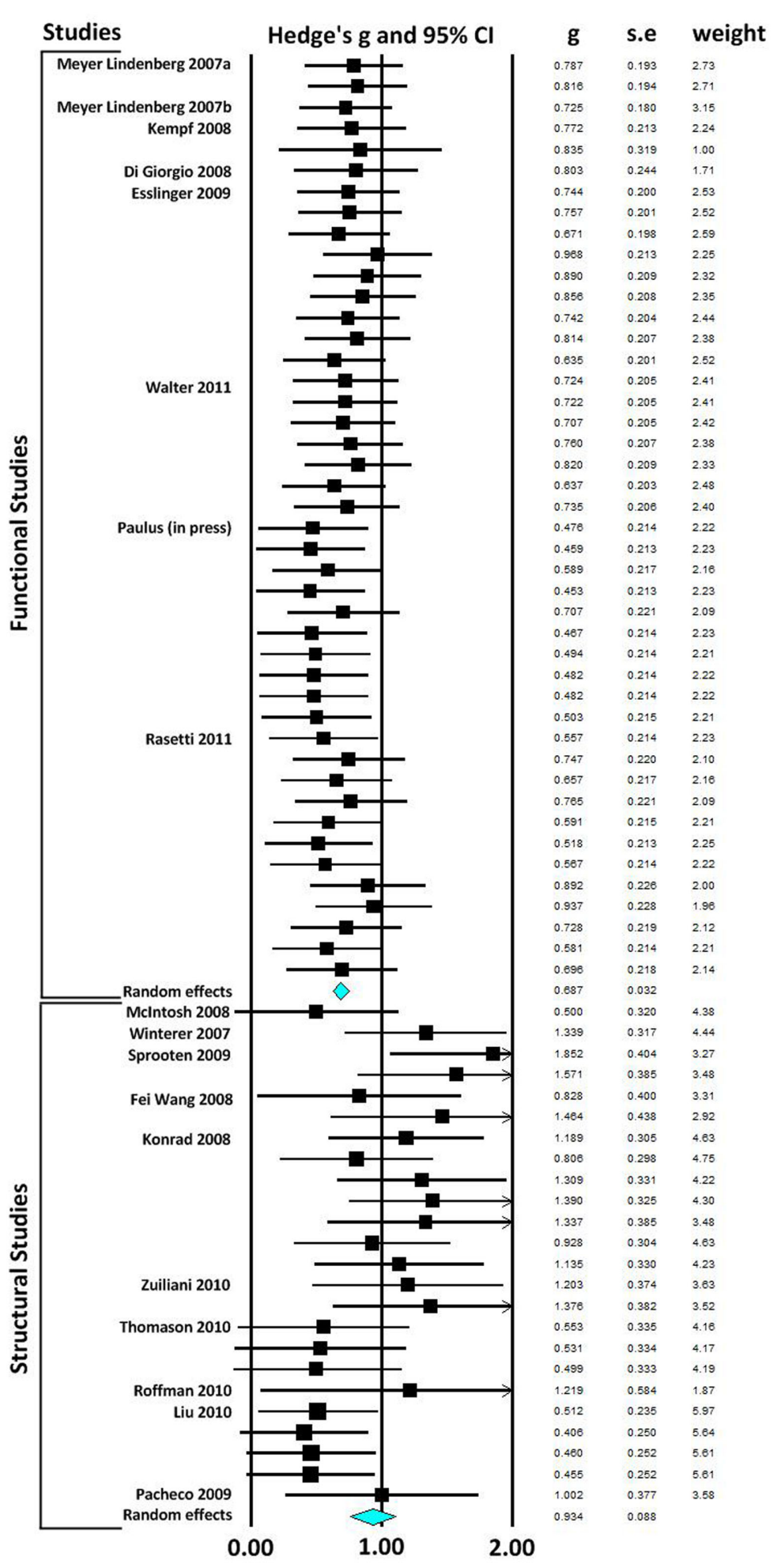

FIGURE 2 | Forest plot reporting Hedges' $\boldsymbol{g}$ and $\mathbf{9 5 \%} \mathbf{C l}$ for each functional and structural connectivity analysis. Cl, confidence interval; g, Hedges' g; SE, standard error.

Other gene variants associated with variation in WM connec- Results for both the MTHFR gene and the 5-HTT gene had large tivity included COMT, MTHFR, 5-HTTLPR, BDNF and DISC1. effect sizes. The MTHFR gene codes for the an enzyme that plays 
a role in the regulation of intracellular methylation reactions and may influence dopamine signaling (Roffman et al., 2008). The hypofunctional 677T variant of this gene has been associated with increased SZ risk (Gilbody et al., 2007). The reuptake of serotonin to the presynaptic neuron for recycling or degradation after serotonin release is regulated by the serotonin transporter (5-HTT). Although the 5-HTTLPR polymorphism has been found to be associated with SZ in a South India population (Vijayan et al., 2009), other genetic association studies have given conflicting results (Rao et al., 1998). The smallest effect size was computed for the effect of a COMT haplotype on left prefrontal WM integrity (Liu et al., 2010). The COMT gene codes for an enzyme that is involved in the degradation of dopamine. Therefore, the evidence from DTI studies investigating the impact of the COMT gene on WM indicates that neurosignalling processes involved in SZ may also impact structural connectivity. Finally, BDNF (Chiang et al., 2011) and DISC1 (Sprooten et al., 2011) which are genes that are crucial for neurodevelopment, were also associated with WM connectivity in SZ. However, as insufficient data was available, effect sizes for these studies were not calculated.

\section{META-ANALYSIS}

The papers included in this review most commonly report a large effect of gene variants on functional and structural connectivity. This result is similar to previous meta-analyses in imaging genetics, which reported large effect sizes of gene variants (i.e., 5-HTTLPR polymorphism and the COMT Val158Met polymorphism) on brain function (Munafo et al., 2008; Mier et al., 2010). This result is also consistent with the intermediate phenotype hypothesis that common SZ risk variants will show small effects on behavior and disease risk, but large effects at the level of the brain (Tost et al., 2011).

When the maximum effect size value for each paper in our meta-analysis was compared between fMRI and DTI studies, no significant difference was found between these measures. As only a small number of studies were obtained, there may be a lack of power to detect such differences. However, examination of effect sizes for all significant effects indicate that structural connectivity studies were associated with overall larger and more variable effect sizes. This suggests that measures of structural connectivity, such as DTI, may be sensitive to a wider range of effects compared to functional connectivity measures, which may only be able to accurately detect large effects. This result may also indicate that structural connectivity is closer to the level of genes than functional connectivity.

\section{LIMITATIONS}

A number of limitations need to be considered in evaluating the findings of the present study. Firstly, many of the studies included in the meta-analysis have examined the effects of polymorphisms that do not have consistent association with SZ phenotypes. This makes it difficult to determine the relevance of these genes for our understanding of SZ pathogenesis (for a review, see MeyerLindenberg, 2010). Secondly, it should also be noted that the sample sizes included in these studies are relatively small and thus, are under powered to detect differences in brain connectivity conferred by individual variants. Due to the interplay between sample size, power, and effect size, smaller studies generally show larger effects in meta-analyses (Sterne et al., 2000) and may lack sufficient power to detect smaller effects. Related to the general issue of sample size, it is important to note that the average sample size of the studies utilizing DTI was smaller than that for the functional studies. As a result, the effect sizes for the structural papers may be over-inflated. However, the results of our meta-analysis suggest that despite smaller samples, the structural imaging studies were associated with a wider range of effects, suggesting that sample size is not the only factor at play here.

Due to the under-representation of publications with negative results, the studies included in this review may not be representative of connectivity research in its entirety, but rather a bias toward only publish papers showing statistically significant results. Therefore, while our effect size findings are calculated on the basis of published effect sizes, it is possible that the true effect sizes are smaller, and to an extent that is unknown. Similarly, it is also unclear to what extent differences in scanning parameters between the studies included in this meta-analysis influenced results. More systematic investigation of these differences will in the future be possible with the accumulation of more studies.

An additional limitation in the studies considered here is that each investigation examined the effects of only one particular variant. However, the true function of these genes may be affected by additive or epistatic interactions with other variants. As such, the results presented in this review may be incomplete without taking these interactions into account (Nicodemus et al., 2010).

Finally, it is probable that these results could be impacted by differences in functional and structural methodological approaches. For example, a number of analysis methods can be employed to measure functional connectivity between brain regions. However, we are not currently aware of the relative strengths and weaknesses of these different approaches. There are also various approaches used to quantify WM connectivity using DTI, which also pose different strengths and limitations (see Jones, 2010 for a review).

\section{CONCLUSIONS AND FUTURE DIRECTIONS}

In a short period of time, imaging genetics has made important progress in delineating genetic effects on neural connectivity. In particular, it has established neural connectivity as a key intermediate phenotype for SZ, which can be used to explore the complex trajectory from genetic risk to clinical symptoms.

Despite the progress that has taken place, we believe important advances can be made in this research field in four key areas. Firstly, future studies should examine the effects of gene variants on neural connectivity in larger sample sizes, as this can provide the extra statistical power that may be necessary to detect smaller effects of these genes. Multi-site research projects, such as the IMAGEN project in Europe, may be particularly suited for compiling imaging and genetic databases of thousands of subjects (Schumann et al., 2010). Secondly, future studies should examine additive and epistatic effects of gene variants on neural connectivity, as these variants are unlikely to be working in isolation. Thirdly, future studies should examine the effects of risk variants in healthy controls and SZ patients as the opposite effects of these genes on connectivity can be found in these different groups (e.g., Prata et al., 2009). 
Finally, future studies could benefit from the novel application of recently developed analysis techniques to imaging genetics. For example, DCM hold potential for constructing models of changing brain interactions that also take into account genetic variation (Meyer-Lindenberg, 2009). Other recent advances include the use of parallel ICA to simultaneously analyze independent components derived from fMRI and genetic data (Liu et al., 2009). For example, Meda et al. (2010) used this technique in a pilot study to identify simultaneous independent components of fMRI data and SNP data, derived from a sample of 35 controls and $31 \mathrm{SZ}$ patients. The authors found correlations between different neural networks and a number of SNPs, including polymorphisms involved in altered dopamine transmission. While the authors only included a small number of SNPs and a small sample size, this research suggests a powerful new approach for future studies examining the effects of SZ risk variants on functional brain networks. Similarly, more advanced DTI techniques could be implemented that use high angular resolution to account for multiple crossing fibers within a single voxel. Such imaging techniques include Q-space approaches and mixture models (Tournier et al., 2011). These models provide mathematical alternatives to the tensor model for the characterization of diffusion processes. Furthermore, Jones (2010) recommends the

\section{REFERENCES}

Braff, D. L., Freedman, R., Schork, N. J., and Gottesman, I. I. (2007). Deconstructing schizophrenia: an overview of the use of endophenotypes in order to understand a complex disorder. Schizophr. Bull. 33, 21.

Breakspear, M., Terry, J. R., Friston, K. J., Harris, A. W., Williams, L. M., Brown, K., Brennan, J., and Gordon, E. (2003). A disturbance of nonlinear interdependence in scalp EEG of subjects with first episode schizophrenia. Neuroimage 20, 466-478.

Buchsbaum, M. S., Schoenknecht, P., Torosjan, Y., Newmark, R., Chu, K. W., Mitelman, S., Brickman, A. M., Shihabuddin, L., Haznedar, M. M., Hazlett, E. A., Ahmed, S., and Tang, C. (2006). Diffusion tensor imaging of frontal lobe white matter tracts in schizophrenia. Ann. Gen. Psychiatry 5, 19.

Callicott, J. H., Bertolino, A., Mattay, V. S., Langheim, F. J., Duyn, J., Coppola, R., Goldberg, T. E., and Weinberger, D. R. (2000). Physiological dysfunction of the dorsolateral prefrontal cortex in schizophrenia revisited. Cereb. Cortex 10, 1078-1092.

Chiang, M. C., Barysheva, M., Toga, A. W., Medland, S. E., Hansell, N. K., James, M. R., McMahon, K. L., de Zubicatay, G. I., Martin, N. G., Wright, M. J., and Thompson, P. M. (2011). BDNF gene effects on brain circuitry replicated in 455 twins. Neuroimage 55, 448-454.
Davis, K. L., Stewart, D. G., Friedman, J. I., Buchsbaum, M., Harvey, P. D., Hof, P. R., Buxbaum, J., and Haroutunian, V. (2003). White matter changes in schizophrenia: evidence for myelin-related dysfunction. Arch. Gen. Psychiatry 60, 443-456.

Di Giorgio, A., Blasi, G., Sambataro, F., Rampino, A., Papazacharias, A., Gambi, F., Romano, R., Caforio, G., Rizzo, M., Latorre, V., Popolizio, T., Kolachana, B., Callicott, J. H., Nardini, M., Weinberger, D. R., and Bertolino, A. (2008). Association of the SerCys DISC1 polymorphism with human hippocampal formation gray matter and function during memory encoding. Eur. J. Neurosci. 28, 2129-2136.

Ellison-Wright, I., and Bullmore, E. (2009). Meta-analysis of diffusion tensor imaging studies in schizophrenia. Schizophr. Res. 108, 3-10.

Esslinger, C., Kirsch, P., Haddad, L., Mier, D., Sauer, C., Erk, S., Schnell, K., Arnold, C., Witt, S. H., Rietschel, M., Cichon, S., Walter, H., and Meyer-Lindenberg, A. (2011). Cognitive state and connectivity effects of the genome-wide significant psychosis variant in ZNF804A. Neuroimage 54, 2514-2523.

Esslinger, C., Walter, H., Kirsch, P., Erk, S., Schnell, K., Arnold, C., Haddad, L., Mier, D., Opitz Von Boberfeld, C., Raab, K., Witt, S. H., Rietschel, M., Cichon, S., and Meyer-Lindenberg, A. (2009). Neural mechanisms of

integration of DTI with other measures of WM such as measures of axon density and myelination that can be acquired using techniques such as magnetization transfer or multicomponent relaxometry.

In conclusion, the present meta-analysis examined the nature and magnitude of effect of SZ risk variants on functional and structural connectivity. Gene variants impacting upon both synaptic plasticity and axonal connectivity have been associated with altered neural connectivity in patients and healthy controls. As such, it is likely that both mechanisms make important contributions to SZ pathogenesis. On average, risk variants exert a large effect on functional and structural connectivity. There is also more variability in the effects of variants on structural connectivity, compared to functional connectivity. While imaging genetics has made considerable progress in the field of neural connectivity in a short period of time, important advances are still to be made. It is hoped that this research will lead to a better understanding of the biological mechanisms mediating genetic risk for SZ, which can then be used to direct novel treatments for the disorder.

\section{ACKNOWLEDGMENTS}

This work was supported by a Science Foundation Ireland research award to Professor Aiden Corvin (SFI08/IN.1/B1916-Corvin).

a genome-wide supported psychosis variant. Science 324, 605.

Fields, R. D. (2008). White matter in learning, cognition and psychiatric disorders. Trends Neurosci. 31, 361-370.

Friston, K. J. (1998). The disconnection hypothesis. Schizophr. Res. 30, 115-125.

Friston, K. J., Buechel, C., Fink, G. R., Morris, J., Rolls, E., and Dolan, R. J. (1997). Psychophysiological and modulatory interactions in neuroimaging. Neuroimage 6, 218-229.

Friston, K. J., and Frith, C. D. (1995) Schizophrenia: a disconnection syndrome? Clin. Neurosci. 3, 89-97.

Friston, K. J., Frith, C. D., Liddle, P. F. and Frackowiak, R. S. (1993). Functional connectivity: the principalcomponent analysis of large (PET) data sets. J. Cereb. Blood Flow Metab. 13, 5-14.

Gilbody, S., Lewis, S., and Lightfoot, T. (2007). Methylenetetrahydrofolate reductase (MTHFR) genetic polymorphisms and psychiatric disorders: a HuGE review. Am. J. Epidemiol. 165, 1-13.

Gottesman, I. I., and Gould, T. D. (2003). The endophenotype concept in psychiatry: etymology and strategic intentions. Am. J. Psychiatry 160, 636-645.

Gustavsson, A., Svensson, M., Jacobi, F., Allgulander, C., Alonso, J., Beghi, E., Dodel, R., Ekman, M., Faravelli, C., Fratiglioni, L., Gannon, B., Jones, D. H., Jennum, P., Jordanova,
A., Jonsson, L., Karampampa, K. Knapp, M., Kobelt, G., Kurth, T., Lieb, R., Linde, M., Ljungcrantz, C., Maercker, A., Melin, B., Moscarelli, M., Musayev, A., Norwood, F., Preisig, M., Pugliatti, M., Rehm, J., Salvador-Carulla, L., Schlehofer, B., Simon, R., Steinhausen, H. C., Stovner, L. J., Vallat, J. M., Den Bergh, P. V., Van Os, J., Vos, P., Xu W., Wittchen, H. U., Jonsson, B., and Olesen, J. (2011). Cost of disorders of the brain in Europe 2010. Eur. Neuropsychopharmacol. 21, 718-779.

Hakak, Y., Walker, J. R., Li, C., Wong, W. H., Davis, K. L., Buxbaum, J. D., Haroutunian, V., and Fienberg, A. A. (2001). Genome-wide expression analysis reveals dysregulation of myelination-related genes in chronic schizophrenia. Proc. Natl. Acad. Sci. U.S.A. 98, 4746-4751.

Harrison, P. J., and Weinberger, D. R. (2005). Schizophrenia genes, gene expression, and neuropathology: on the matter of their convergence. Mol. Psychiatry 10, 40-68; image 45.

Javitt, D. C. (2010). Glutamatergic theories of schizophrenia. Isr. J. Psychiatry Relat. Sci. 47, 4-16.

Jones, D. K. (2008). Studying connections in the living human brain with diffusion MRI. Cortex 44, 936-952.

Jones, D. K. (2010). Challenges and limitations of quantifying brain connectivity in vivo with diffusion MRI Imaging 2, 341-355. 
Kegeles, L. S., Abi-Dargham, A., Zea-Ponce, Y., Rodenhiser-Hill, J., Mann, J. J., Van Heertum, R. L., Cooper, T. B., Carlsson, A., and Laruelle, M. (2000). Modulation of amphetamine-induced striatal dopamine release by ketamine in humans: implications for schizophrenia. Biol. Psychiatry 48, 627-640.

Kempf, L., Nicodemus, K. K., Kolachana, B., Vakkalanka, R., Verchinski, B. A., Egan, M. F., Straub, R. E., Mattay, V. A., Callicott, J. H., Weinberger, D. R., and MeyerLindenberg, A. (2008). Functional polymorphisms in PRODH are associated with risk and protection for schizophrenia and fronto-striatal structure and function. PLoS Genet. 4, e1000252.

Konrad, A., Vucurevic, G., Musso, F., Stoeter, P., Dahmen, N., and Winterer, G. (2009). ErbB4 genotype predicts left frontotemporal structural connectivity in human brain. Neuropsychopharmacology 34 , 641-650.

Konrad, A., and Winterer, G. (2008). Disturbed structural connectivity in schizophrenia primary factor in pathology or epiphenomenon? Schizophr. Bull. 34, 72-92.

Kubicki, M., Mccarley, R., Westin, C. F., Park, H. J., Maier, S., Kikinis, R., Jolesz, F. A., and Shenton, M. E. (2007). A review of diffusion tensor imaging studies in schizophrenia. J. Psychiatr. Res. 41, 15-30.

Lawrie, S. M., Buechel, C., Whalley, H. C., Frith, C. D., Friston, K. J., and Johnstone, E. C. (2002). Reduced frontotemporal functional connectivity in schizophrenia associated with auditory hallucinations. Biol. Psychiatry 51, 1008-1011.

Lewis, D. A., and Lieberman, J. A. (2000). Catching up on schizophrenia: natural history and neurobiology. Neuron 28, 325-334.

Liu, B., Li, J., Yu, C., Li, Y., Liu, Y., Song, M., Fan, M., Li, K., and Jiang, T. (2010). Haplotypes of catechol-O-methyltransferase modulate intelligence-related brain white matter integrity. Neuroimage 50, 243-249.

Liu, J., Pearlson, G., Windemuth, A., Ruano, G., Perrone-Bizzozero, N. I., and Calhoun, V. (2009). Combining fMRI and SNP data to investigate connections between brain function and genetics using parallel ICA. Hum. Brain Mapp. 30, 241-255.

McIntosh, A. M., Moorhead, T. W., Job, D., Lymer, G. K., Munoz Maniega, S., Mckirdy, J., Sussmann, J. E., Baig, B. J., Bastin, M. E., Porteous, D., Evans,
K. L., Johnstone, E. C., Lawrie, S. M., and Hall, J. (2008). The effects of a neuregulin 1 variant on white matter density and integrity. Mol. Psychiatry 13, 1054-1059.

Meda, S. A., Jagannathan, K., Gelernter, J., Calhoun, V. D., Liu, J., Stevens, M. C., and Pearlson, G. D. (2010). A pilot multivariate parallel ICA study to investigate differential linkage between neural networks and genetic profiles in schizophrenia. Neuroimage 53, 1007-1015.

Meyer-Lindenberg, A. (2009). Neural connectivity as an intermediate phenotype: brain networks under genetic control. Hum. Brain Mapp. 30, 1938-1946.

Meyer-Lindenberg, A. (2010). From maps to mechanisms through neuroimaging of schizophrenia. Nature 468, 194-202.

Meyer-Lindenberg, A., Poline, J. B., Kohn, P. D., Holt, J. L., Egan, M. F., Weinberger, D. R., and Berman, K. F. (2001). Evidence for abnormal cortical functional connectivity during working memory in schizophrenia. Am. J. Psychiatry 158, 1809-1817.

Meyer-Lindenberg, A., Straub, R. E., Lipska, B. K., Verchinski, B. A., Goldberg, T., Callicott, J. H., Egan, M. F., Huffaker, S. S., Mattay, V. S., Kolachana, B., Kleinman, J. E., and Weinberger, D. R. (2007). Genetic evidence implicating DARPP-32 in human frontostriatal structure, function, and cognition. J. Clin. Invest. 117, 672-682.

Meyer-Lindenberg, A. S., Olsen, R. K., Kohn, P. D., Brown, T., Egan, M. F., Weinberger, D. R., and Berman, K. F. (2005). Regionally specific disturbance of dorsolateral prefrontalhippocampal functional connectivity in schizophrenia. Arch. Gen. Psychiatry 62, 379-386.

Mier, D., Kirsch, P., and MeyerLindenberg, A. (2010). Neural substrates of pleiotropic action of genetic variation in COMT: a meta-analysis. Mol. Psychiatry 15, 918-927.

Munafo, M. R., Brown, S. M., and Hariri, A. R. (2008). Serotonin transporter (5-HTTLPR) genotype and amygdala activation: a meta-analysis. Biol. Psychiatry 63, 852-857.

Nallasamy, N., and Tsao, D. Y. (2011). Functional connectivity in the brain: effects of anesthesia. Neuroscientist. $17,94-106$.

Nicodemus, K. K., Law, A. J., Radulescu, E., Luna, A., Kolachana, B., Vakkalanka, R., Rujescu, D., Giegling, I., Straub, R. E., Mcgee, K., Gold, B., Dean, M., Muglia, P.,
Callicott, J. H., Tan, H. Y., and Weinberger, D. R. (2010). Biological validation of increased schizophrenia risk with NRG1, ERBB4, and AKT1 epistasis via functional neuroimaging in healthy controls. Arch. Gen. Psychiatry 67, 991-1001.

Norton, N., Moskvina, V., Morris, D. W., Bray, N. J., Zammit, S., Williams, N. M., Williams, H. J., Preece, A. C., Dwyer, S., Wilkinson, J. C., Spurlock, G., Kirov, G., Buckland, P. Waddington, J. L., Gill, M., Corvin, A. P., Owen, M. J., and O'Donovan, M. C. (2006). Evidence that interaction between neuregulin 1 and its receptor erbB4 increases susceptibility to schizophrenia. Am. J. Med. Genet. B Neuropsychiatr. Genet. 141B, 96-101.

O’Donovan, M. C., Craddock, N., Norton, N., Williams, H., Peirce, T. Moskvina, V., Nikolov, I., Hamshere, M., Carroll, L., Georgieva, L., Dwyer, S., Holmans, P., Marchini, J. L., Spencer, C. C., Howie, B., Leung, H. T., Hartmann, A. M., Moller, H. J., Morris, D. W., Shi, Y., Feng, G., Hoffmann, P., Propping, P., Vasilescu, C., Maier, W., Rietschel, M., Zammit, S., Schumacher, J., Quinn, E. M., Schulze, T. G., Williams, N. M., Giegling, I., Iwata, N., Ikeda, M., Darvasi, A., Shifman, S., He, L., Duan, J., Sanders, A. R., Levinson, D. F., Gejman, P. V., Cichon, S. Nothen, M. M., Gill, M., Corvin, A. Rujescu, D., Kirov, G., Owen, M. J. Buccola, N. G., Mowry, B. J., Freedman, R., Amin, F., Black, D. W., Silverman, J. M., Byerley, W. F., and Cloninger, C. R. (2008). Identification of loci associated with schizophrenia by genome-wide association and follow-up. Nat. Genet. 40, 1053-1055.

Ogawa, S., Lee, T. M., Kay, A. R., and Tank, D. W. (1990). Brain magnetic resonance imaging with contrast dependent on blood oxygenation. Proc. Natl. Acad. Sci. U.S.A. 87, 9868-9872.

Pacheco, J., Beevers, C. G., Benavides, C., Mcgeary, J., Stice, E., and Schnyer, D. M. (2009). Frontallimbic white matter pathway associations with the serotonin transporter gene promoter region (5HTTLPR) polymorphism. J. Neurosci. 29, 6229-6233.

Paulus, F. M., Krach, S., Bedenbender, J., Pyka, M., Sommer, J., Krug, A. Knake, S., Nothen, M. M., Witt, S. H., Rietschel, M., Kircher, T., and Jansen, A. (2011). Partial support for ZNF804A genotype-dependent alterations in prefrontal connectivity. Hum. Brain Mapp.
Perez-Iglesias, R., Tordesillas-Gutierrez, D., Barker, G. J., Mcguire, P. K., RoizSantianez, R., Mata, I., De Lucas, E. M., Quintana, F., Vazquez-Barquero, J. L., and Crespo-Facorro, B. (2010). White matter defects in first episode psychosis patients: a voxelwise analysis of diffusion tensor imaging. Neuroimage 49, 199-204.

Pezawas, L., Meyer-Lindenberg, A., Drabant, E. M., Verchinski, B. A. Munoz, K. E., Kolachana, B. S., Egan, M. F., Mattay, V. S., Hariri, A. R., and Weinberger, D. R. (2005). 5-HTTLPR polymorphism impacts human cingulate-amygdala interactions: a genetic susceptibility mechanism for depression. Nat. Neurosci. $8,828-834$

Pitt, D., Nagelmeier, I. E., Wilson, H. C. and Raine, C. S. (2003). Glutamate uptake by oligodendrocytes: Implications for excitotoxicity in multiple sclerosis. Neurology 61, 1113-1120.

Prata, D. P., Mechelli, A., Fu, C. H., Picchioni, M., Kane, F., Kalidindi, S., Mcdonald, C., Howes, O., Kravariti, E., Demjaha, A., Toulopoulou, T., Diforti, M., Murray, R. M., Collier, D. A., and Mcguire, P. K. (2009). Opposite effects of catecholO-methyltransferase Val158Met on cortical function in healthy subjects and patients with schizophrenia. Biol. Psychiatry 65, 473-480.

Rao, D., Jonsson, E. G., Paus, S., Ganguli, R., Nothen, M., and Nimgaonkar, V. L. (1998). Schizophrenia and the serotonin transporter gene. Psychiatr. Genet. 8, 207-212.

Rasetti, R., Sambataro, F., Chen, Q., Callicott, J. H., Mattay, V. S., and Weinberger, D. R. (2011). Altered Cortical Network Dynamics: A Potential Intermediate Phenotype for Schizophrenia and Association With ZNF804A. Arch. Gen. Psychiatry 68, 1207-1217.

Roffman, J. L., Brohawn, D. G., Friedman, J. S., Dyckman, K. A., Thakkar K. N., Agam, Y., Vangel, M. G., Goff, D. C., and Manoach, D. S. (2011) MTHFR $677 \mathrm{C}>\mathrm{T}$ effects on anterior cingulate structure and function during response monitoring in schizophrenia: a preliminary study. Brain Imaging Behav. 5, 65-75.

Roffman, J. L., Weiss, A. P., Purcell, S., Caffalette, C. A., Freudenreich, O., Henderson, D. C., Bottiglieri, T., Wong, D. H., Halsted, C. H., and Goff, D. C. (2008). Contribution of methylenetetrahydrofolate reductase (MTHFR) polymorphisms to negative symptoms in schizophrenia. Biol. Psychiatry 63, 42-48.

Schumann, G., Loth, E., Banaschewski, T., Barbot, A., Barker, G., Buchel, 
C., Conrod, P. J., Dalley, J. W., Flor, H., Gallinat, J., Garavan, H., Heinz, A., Itterman, B., Lathrop, M., Mallik, C., Mann, K., Martinot, J. L., Paus, T., Poline, J. B., Robbins, T. W., Rietschel, M., Reed, L., Smolka, M., Spanagel, R., Speiser, C., Stephens, D. N., Strohle, A., and Struve, M. (2010). The IMAGEN study: reinforcement-related behaviour in normal brain function and psychopathology. Mol. Psychiatry 15, 1128-1139.

Selemon, L. D., and Goldman-Rakic, P. S. (1999). The reduced neuropil hypothesis: a circuit based model of schizophrenia. Biol. Psychiatry 45, 17-25.

Sigurdsson, T., Stark, K. L., Karayiorgou, M., Gogos, J. A., and Gordon, J. A. (2010). Impaired hippocampalprefrontal synchrony in a genetic mouse model of schizophrenia. Nature 464, 763.

Sprooten, E., Lymer, G. K., Munoz Maniega, S., Mckirdy, J., Clayden, J. D., Bastin, M. E., Porteous, D., Johnstone, E. C., Lawrie, S. M., Hall, J., and Mcintosh, A. M. (2009). The relationship of anterior thalamic radiation integrity to psychosis risk associated neuregulin-1 variants. Mol. Psychiatry 14, 237-238, 233.

Sprooten, E., Sussman, J. E., Moorhear, T. W., Whalley, H. C., ffrenchConstant, C., Blumberg, H. P., Bastin, M. E., Hall, J., Lawrie, S. M., and McIntosh, A. M. (2011). Association of white matter imtegrity with genetic variation in an exonic DISC1 SNP. Mol. Psychiatry 1-2.

Stephan, K. E., Friston, K. J., and Frith, C. D. (2009). Dysconnection in schizophrenia: from abnormal synaptic plasticity to failures of self-monitoring. Schizophr. Bull. 35 , 509-527.

Sterne, J. A., Gavaghan, D., and Egger, M. (2000). Publication and related bias in meta-analysis: power of statistical tests and prevalence in the literature. J. Clin. Epidemiol. 53, 1119-1129.

Sullivan, P. F., Kendler, K. S., and Neale, M. C. (2003). Schizophrenia as a complex trait: evidence from a metaanalysis of twin studies. Arch. Gen. Psychiatry 60, 1187-1192.

Takahashi, N., Sakurai, T., Davis, K. L., and Buxbaum, J. D. (2011). Linking oligodendrocyte and myelin dysfunction to neurocircuitry abnormalities in schizophrenia. Prog. Neurobiol. 93, 13-24.

Tanaka, H., Ma, J., Tanaka, K. F., Takao, K., Komada, M., Tanda, K., Suzuki, A., Ishibashi, T., Baba, H., Isa, T., Shigemoto, R., Ono, K., Miyakawa, T., and Ikenaka, K. (2009). Mice with altered myelin proteolipid protein gene expression display cognitive deficits accompanied by abnormal neuron-glia interactions and decreased conduction velocities. $J$. Neurosci. 29, 8363-8371.

Thomason, M. E., Dougherty, R. F., Colich, N. L., Perry, L. M., Rykhlevskaia, E. I., Louro, H. M., Hallmayer, J. F., Waugh, C. E., Bammer, R., Glover, G. H., and Gotlib, I. H. (2010). COMT genotype affects prefrontal white matter pathways in children and adolescents. Neuroimage 53, 926-934.

Tost, H., Bilek, E., and MeyerLindenberg, A. (2011). Brain connectivity in psychiatric imaging genetics. Neuroimage. doi: 10.1016/j.neuroimage.2011.11.007
Tournier, J. D., Mori, S., and Leemans, A. (2011). Diffusion tensor imaging and beyond. Magn. Reson. Med. 65, 1532-1556.

Umbricht, D., Schmid, L., Koller, R., Vollenweider, F. X., Hell, D., and Javitt, D. C. (2000). Ketamineinduced deficits in auditory and visual context-dependent processing in healthy volunteers: implications for models of cognitive deficits in schizophrenia. Arch. Gen. Psychiatry 57, 1139-1147.

Vijayan, N. N., Iwayama, Y., Koshy, L. V., Natarajan, C., Nair, C., Allencherry, P. M., Yoshikawa, T., and Banerjee, M. (2009). Evidence of association of serotonin transporter gene polymorphisms with schizophrenia in a South Indian population. J. Hum. Genet. 54, 538-542.

Walter, H., Schnell, K., Erk, S., Arnold, C., Kirsch, P., Esslinger, C., Mier D., Schmitgen, M. M., Rietschel, M., Witt, S. H., Nothen, M. M., Cichon, S., and Meyer-Lindenberg, A. (2011). Effects of a genome-wide supported psychosis risk variant on neural activation during a theoryof-mind task. Mol. Psychiatry 16, 462-470.

Wang, F., Jiang, T., Sun, Z., Teng, S. L. Luo, X., Zhu, Z., Zang, Y., Zhang, H., Yue, W., Qu, M., Lu, T., Hong, N., Huang, H., Blumberg, H. P., and Zhang, D. (2009). Neuregulin 1 genetic variation and anterior cingulum integrity in patients with schizophrenia and healthy controls. JPsychiatry Neurosci 34, 181-186.

Winterer, G., Konrad, A., Vucurevic, G., Musso, F., Stoeter, P., and Dahmen, N. (2008). Association of $5^{\prime}$ end neuregulin-1 (NRG1) gene variation with subcortical medial frontal microstructure in humans. Neuroimage $40,712-718$.

Witthaus, H., Brune, M., Kaufmann, C., Bohner, G., Ozgurdal, S., Gudlowski, Y., Heinz, A., Klingebiel, R., and Juckel, G. (2008). White matter abnormalities in subjects at ultra high-risk for schizophrenia and first-episode schizophrenic patients. Schizophr. Res. 102, 141-149.

Zuliani, R., Moorhead, T. W., Bastin, M. E., Johnstone, E. C., Lawrie, S. M., Brambilla, P., O’Donovan, M. C., Owen, M. J., Hall, J., and Mcintosh, A. M. (2011). Genetic variants in the ErbB4 gene are associated with white matter integrity. Psychiatry Res. 191, 133-137.

Conflict of Interest Statement: The authors declare that the research was conducted in the absence of any commercial or financial relationships that could be construed as a potential conflict of interest.

Received: 02 December 2011; accepted: 22 February 2012; published online: 09 March 2012.

Citation: Mothersill O, Kelly S, Rose EJ and Donohoe G (2012) The effects of psychosis risk variants on brain connectivity: a review. Front. Psychiatry 3:18. doi: 10.3389/fpsyt.2012.00018

This article was submitted to Frontiers in Molecular Psychiatry, a specialty of Frontiers in Psychiatry.

Copyright (C) 2012 Mothersill, Kelly, Rose and Donohoe. This is an open-access article distributed under the terms of the Creative Commons Attribution Non Commercial License, which permits noncommercial use, distribution, and reproduction in other forums, provided the original authors and source are credited. 